\title{
An operator method for telegraph partial differential and difference equations
}

\author{
Allaberen Ashyralyev ${ }^{1 *}$ and Mahmut Modanli ${ }^{2}$
}

"Correspondence: aashyr@fatih.edu.tr

${ }^{1}$ Department of Mathematics, Fatih University, Istanbul, 34500, Turkey

Full list of author information is

available at the end of the article

\begin{abstract}
The Cauchy problem for abstract telegraph equations $\frac{d^{2} u(t)}{d t^{2}}+\alpha \frac{d u(t)}{d t}+A u(t)+\beta u(t)=$ $f(t)(0 \leq t \leq T), u(0)=\varphi, u^{\prime}(0)=\psi$ in a Hilbert space $H$ with the self-adjoint positive definite operator $A$ is studied. Stability estimates for the solution of this problem are established. The first and second order of accuracy difference schemes for the approximate solution of this problem are presented. Stability estimates for the solution of these difference schemes are established. In applications, two mixed problems for telegraph partial differential equations are investigated. The methods are illustrated by numerical examples.
\end{abstract}

Keywords: telegraph equations; Cauchy problem; Hilbert space; difference schemes; stability

\section{Introduction}

Hyperbolic partial differential equations arise in many branches of science and engineering, e.g., electromagnetic, electrodynamics, thermodynamics, hydrodynamics, elasticity, fluid dynamics, wave propagation, materials science. In numerical methods for solving these equations, the problem of stability has received a great deal of importance and attention. Specially, a suitable model for analyzing the stability is provided by a proper unconditionally absolutely stable difference scheme with an unbounded operator. The role played by the positivity property of differential and difference operators in Hilbert and Banach spaces in the study of various properties of boundary value problems for partial differential equations, of stability of difference schemes for partial differential equations, and of summation Fourier series is well known (see [1-9]).

The method of operators as a tool for the investigation of the solution of local and nonlocal problems to hyperbolic differential equations in Hilbert and Banach spaces, has been systematically developed by several authors (see, e.g., [1-3, 10, 11]).

The telegraph hyperbolic partial differential equation is important for modeling several relevant problems such as signal analysis, wave propagation, random walk theory [12-14]. To deal with the equation, various mathematical methods have been proposed for obtaining exact and approximate analytic solutions. For instances, Dehghan and Shokri proposed a new numerical scheme based on radial based function method (Kansa's method) [15]. Gao and Chi developed a numerical algorithm for the solution of nonlinear telegraph equations [16]. Biazar et al. applied the variational iteration method to obtain an approximate of the telegraph equations [17]. Saadatmandi and Dehghan used the Chebyshev 
tau method in numerically solving the telegraph equation [18]. Koksal computed numerical solutions the telegraph equations arising in transmission lines [19]. Twizell used the explicit difference method for the wave equation with extended stability range [20]. Finally, Ashyralyev and Akat applied the difference method for the approximate solution of stochastic hyperbolic and stochastic telegraph equations [21-23].

In the present paper, we consider a Cauchy problem for telegraph equations

$$
\left\{\begin{array}{l}
\frac{d^{2} u(t)}{d t^{2}}+\alpha \frac{d u(t)}{d t}+A u(t)+\beta u(t)=f(t) \quad(0 \leq t \leq T) \\
u(0)=\varphi, \quad u^{\prime}(0)=\psi
\end{array}\right.
$$

in a Hilbert space $H$ with a self-adjoint positive definite operator $A$ and $A \geq \delta I$. Here $\delta>0$, $\alpha>0$ and

$$
\beta+\delta \geq \frac{\alpha^{2}}{4}
$$

A function $u(t)$ is called a solution of the problem (1) if the following conditions are satisfied:

i. $u(t)$ is twice continuously differentiable on the segment $[0, T]$. The derivatives at the endpoints of the segment are understood as the appropriate unilateral derivatives.

ii. The element $u(t)$ belongs to $D(A)$ for all $t \in[0, T]$ and the function $A u(t)$ is continuous on the segment $[0, T]$.

iii. $u(t)$ satisfies the equation and initial conditions (1).

The paper is organized as follows. Section 1 is an introduction where we provide the definition of the solution of the Cauchy problem (1). In Section 2, stability estimates for the solution of this problem are established. In applications, two mixed problems for telegraph partial differential equations are investigated. In Section 3, the difference schemes of the first and second order of accuracy for the approximate solution of problem (1) are presented. Stability estimates for the solution of these difference schemes are established. In applications, stability estimates for the solution of difference schemes for the two mixed problems for telegraph partial differential equations are established. In Section 4, the methods are illustrated by numerical examples. Section 5 is for our conclusion.

\section{The main theorem on stability}

Let $\{c(t), t \geq 0\}$ be a strongly continuous cosine operator-function defined by the formula

$$
c(t)=\frac{e^{i t B^{1 / 2}}+e^{-i t B^{1 / 2}}}{2} .
$$

Then, from the definition of the sine operator-function $s(t)$,

$$
s(t) u=\int_{0}^{t} c(s) u d s
$$

it follows that

$$
s(t)=B^{-1 / 2} \frac{e^{i t B^{1 / 2}}-e^{-i t B^{1 / 2}}}{2 i} .
$$


Here $B=A+\left(\beta-\frac{\alpha^{2}}{4}\right) I$. It is easy to check under the assumption (2) that the problem (1) for a telegraph equation has a unique mild solution given by the formula

$$
u(t)=e^{-\frac{\alpha}{2} t} c(t) \varphi+\frac{\alpha}{2} e^{-\frac{\alpha}{2} t} s(t) \varphi+e^{-\frac{\alpha}{2} t} s(t) \psi+\int_{0}^{t} e^{-\frac{\alpha}{2}(t-z)} s(t-z) f(z) d z
$$

In fact, explicitly (1) can be rewritten as the equivalent initial-value problem for a system of first-order differential equations

$$
\left\{\begin{array}{l}
u^{\prime}(t)+\frac{\alpha}{2} v(t)+i B^{\frac{1}{2}} u(t)=z(t) \quad(0 \leq t \leq T) \\
u(0)=u_{0}, \quad u^{\prime}(0)=u_{0}^{\prime} \\
z^{\prime}(t)+\frac{\alpha}{2} z(t)-i B^{\frac{1}{2}} z(t)=f(t) .
\end{array}\right.
$$

Integrating these, now we get

$$
\left\{\begin{array}{l}
u(t)=e^{-\left(\frac{\alpha}{2}+i B^{\frac{1}{2}}\right) t} u(0)+\int_{0}^{t} e^{-\left(\frac{\alpha}{2}+i B^{\frac{1}{2}}\right)(t-s)} z(s) d s \\
z(t)=e^{-\left(\frac{\alpha}{2}-i B^{\frac{1}{2}}\right) t} z(0)+\int_{0}^{t} e^{-\left(\frac{\alpha}{2}-i B^{\frac{1}{2}}\right)(t-s)} f(s) d s
\end{array}\right.
$$

Applying the initial condition $z(0)=u^{\prime}(0)+\left(\frac{\alpha}{2}+i B^{\frac{1}{2}}\right) u(0)$, we get

$$
\begin{aligned}
u(t)= & e^{-\left(\frac{\alpha}{2}+i B^{\frac{1}{2}}\right) t} u(0)+\int_{0}^{t} e^{-\left(\frac{\alpha}{2}+i B^{\frac{1}{2}}\right)(t-s)} \int_{0}^{s} e^{-\left(\frac{\alpha}{2}-i B^{\frac{1}{2}}\right)(s-p)} f(p) d p d s \\
& +\int_{0}^{t} e^{-\left(\frac{\alpha}{2}+i B^{\frac{1}{2}}\right)(t-s)} e^{-\left(\frac{\alpha}{2}-i B^{\frac{1}{2}}\right) s} d s\left(u^{\prime}(0)+\left(\frac{\alpha}{2}+i B^{\frac{1}{2}}\right) u(0)\right) .
\end{aligned}
$$

By an interchange of the order of integration, we can write

$$
\begin{aligned}
u(t)= & {\left[e^{-\left(\frac{\alpha}{2}+i B^{\frac{1}{2}}\right) t}+\left(\frac{\alpha}{2}+i B^{\frac{1}{2}}\right) \int_{0}^{t} e^{-\left(\frac{\alpha}{2}+i B^{\frac{1}{2}}\right)(t-s)} e^{-\left(\frac{\alpha}{2}-i B^{\frac{1}{2}}\right) s} d s\right] u(0) } \\
& +\int_{0}^{t} e^{-\left(\frac{\alpha}{2}+i B^{\frac{1}{2}}\right)(t-s)} e^{-\left(\frac{\alpha}{2}-i B^{\frac{1}{2}}\right) s} d s u^{\prime}(0) \\
& +\int_{0}^{t} e^{-\frac{\alpha}{2}(t-s)} B^{-\frac{1}{2}} \frac{e^{i(t-s) B^{\frac{1}{2}}}-e^{-i(t-s) B^{\frac{1}{2}}}}{2 i} f(s) d s \\
= & e^{-\frac{\alpha}{2} t}\left[\frac{e^{i t B^{\frac{1}{2}}}+e^{-i t B^{\frac{1}{2}}}}{2}+\frac{\alpha}{2} B^{-\frac{1}{2}} \frac{e^{t i B^{\frac{1}{2}}}-e^{-i t B^{\frac{1}{2}}}}{2 i}\right] u(0) \\
& +e^{-\frac{\alpha}{2} t}\left[B^{-\frac{1}{2}} \frac{e^{i t B^{\frac{1}{2}}}-e^{-i t B^{\frac{1}{2}}}}{2 i}\right] u^{\prime}(0) \\
& +\int_{0}^{t} e^{-\frac{\alpha}{2}(t-s)} B^{-\frac{1}{2}} \frac{e^{i(t-s) B^{\frac{1}{2}}}-e^{-i(t-s) B^{\frac{1}{2}}}}{2 i} f(s) d s .
\end{aligned}
$$

Thus, by the definitions of $B^{\frac{1}{2}}, c(t)$, and $s(t)$ we obtain (3). We will prove the following main theorem on the continuous dependence of the solution on the given data. 
Theorem 2.1 Suppose that $\varphi \in D(A), \psi \in D\left(A^{\frac{1}{2}}\right)$ and $f(t)$ is a continuously differentiable function on $[0, T]$ and the assumption (2) holds. Then there is a unique solution of problem (1) and the stability inequalities

$$
\begin{aligned}
& \max _{0 \leq t \leq T}\|u(t)\|_{H} \leq M\left[\|\varphi\|_{H}+\left\|A^{-1 / 2} \psi\right\|_{H}+\max _{0 \leq t \leq T}\left\|A^{-1 / 2} f(t)\right\|_{H}\right], \\
& \max _{0 \leq t \leq T}\left\|\frac{d u(t)}{d t}\right\|_{H}+\max _{0 \leq t \leq T}\left\|A^{1 / 2} u(t)\right\|_{H} \\
& \quad \leq M\left[\left\|A^{1 / 2} \varphi\right\|_{H}+\|\psi\|_{H}+\max _{0 \leq t \leq T}\|f(t)\|_{H}\right], \\
& \max _{0 \leq t \leq T}\left\|\frac{d^{2} u(t)}{d t^{2}}\right\|_{H}+\max _{0 \leq t \leq T}\|A u(t)\|_{H} \\
& \quad \leq M\left[\|A \varphi\|_{H}+\left\|A^{1 / 2} \psi\right\|_{H}+\|f(0)\|_{H}+\max _{0 \leq t \leq T}\left\|f^{\prime}(t)\right\|_{H}\right]
\end{aligned}
$$

hold, where $M$ does not depend on $\varphi, \psi$, and $f(t)$.

Proof Using (3), $A \geq \delta I$, and the following estimates:

$$
\left\{\begin{array}{l}
\|c(t)\|_{H \rightarrow H} \leq 1, \quad\left\|B^{\frac{1}{2}} s(t)\right\|_{H \rightarrow H} \leq 1, \quad\left|e^{-\frac{\alpha}{2} t}\right| \leq 1, \\
\left\|B^{-1 / 2} \varphi\right\|_{H} \leq \frac{1}{\sqrt{\delta}}\|\varphi\|_{H}, \quad\left\|A^{1 / 2} B^{-\frac{1}{2}}\right\|_{H \rightarrow H} \leq M(\delta),
\end{array}\right.
$$

we can write the following inequalities:

$$
\begin{gathered}
\|u(t)\|_{H} \leq\|c(t)\|_{H \rightarrow H}\left|e^{-\frac{\alpha}{2} t}\right|\|\varphi\|_{H}+\left\|B^{\frac{1}{2}} s(t)\right\|_{H \rightarrow H}\left\|A^{1 / 2} B^{-\frac{1}{2}}\right\|_{H \rightarrow H} \\
\left|\frac{\alpha}{2 e^{\frac{\alpha}{2}} t}\right|\left\|A^{-1 / 2} \varphi\right\|_{H}+\left\|B^{\frac{1}{2}} s(t)\right\|_{H \rightarrow H}\left\|A^{1 / 2} B^{-\frac{1}{2}}\right\|_{H \rightarrow H}\left|e^{-\frac{\alpha}{2} t}\right|\left\|A^{-1 / 2} \psi\right\|_{H} \\
\quad+\int_{0}^{t}\left\|B^{\frac{1}{2}} s(t-s)\right\|_{H \rightarrow H}\left\|A^{1 / 2} B^{-\frac{1}{2}}\right\|_{H \rightarrow H}\left\|A^{-1 / 2} f(s)\right\|_{H} d s \\
\leq M_{1}(\delta)\left[\|\varphi\|_{H}+\left\|A^{-1 / 2} \psi\right\|_{H}+\max _{0 \leq t \leq T}\left\|A^{-1 / 2} f(t)\right\|_{H}\right]
\end{gathered}
$$

for any $t \in[0, T]$. Then we obtain

$$
\max _{0 \leq t \leq T}\|u(t)\|_{H} \leq M_{1}(\delta)\left[\|\varphi\|_{H}+\left\|A^{-1 / 2} \psi\right\|_{H}+\max _{0 \leq t \leq T}\left\|A^{-1 / 2} f(t)\right\|_{H}\right] .
$$

Applying $A^{\frac{1}{2}}$ to (3) and using the estimate for (8), in a similar manner, we get

$$
\begin{aligned}
\left\|A^{\frac{1}{2}} u(t)\right\|_{H} \leq & \|c(t)\|_{H \rightarrow H}\left|e^{-\frac{\alpha}{2} t}\right|\left\|A^{\frac{1}{2}} \varphi\right\|_{H} \\
& +\left\|B^{\frac{1}{2}} s(t)\right\|_{H \rightarrow H}\left\|A^{1 / 2} B^{-\frac{1}{2}}\right\|_{H \rightarrow H}\left|\frac{\alpha}{2 e^{\frac{\alpha}{2}} t}\right|\|\varphi\|_{H} \\
& +\left\|B^{\frac{1}{2}} s(t)\right\|_{H \rightarrow H}\left\|A^{1 / 2} B^{-\frac{1}{2}}\right\|_{H \rightarrow H}\left|e^{-\frac{\alpha}{2} t}\right|\|\psi\|_{H} \\
& +\int_{0}^{t}\left\|A^{1 / 2} B^{-\frac{1}{2}}\right\|_{H \rightarrow H}\left\|B^{\frac{1}{2}} s(t-s)\right\|_{H \rightarrow H}\|f(s)\|_{H} d s \\
\leq & M_{2}(\delta)\left[\left\|A^{\frac{1}{2}} \varphi\right\|_{H}+\|\psi\|_{H}+\max _{0 \leq t \leq T}\|f(t)\|_{H}\right]
\end{aligned}
$$


for any $t \in[0, T]$. Then we get

$$
\max _{0 \leq t \leq T}\left\|A^{\frac{1}{2}} u(t)\right\|_{H} \leq M_{2}(\delta)\left[\left\|A^{\frac{1}{2}} \varphi\right\|_{H}+\|\psi\|_{H}+\max _{0 \leq t \leq T}\|f(t)\|_{H}\right] .
$$

Now, we obtain an estimate for $\|A u(t)\|_{H}$. Applying $A$ to (3) and using an integration by parts, we can write the formula

$$
\begin{aligned}
A u(t) e^{\frac{\alpha}{2} t}= & c(t) A \varphi+\frac{\alpha}{2} A^{\frac{1}{2}} s(t) A^{\frac{1}{2}} \varphi+A^{\frac{1}{2}} s(t) A^{\frac{1}{2}} \psi \\
& +A B^{-1}\left[f(T)-c(t) f(0)-\int_{0}^{t} c(t-s) f^{\prime}(s) d s\right]
\end{aligned}
$$

Using the last formula and estimates (8), we obtain

$$
\begin{aligned}
\|A u(t)\|_{H} \leq & \|c(t)\|_{H \rightarrow H}\left|e^{-\frac{\alpha}{2} t}\right|\|A \varphi\|_{H} \\
& +\left\|B^{\frac{1}{2}} s(t)\right\|_{H \rightarrow H}\left\|A^{1 / 2} B^{-\frac{1}{2}}\right\|_{H \rightarrow H}\left|\frac{\alpha}{2 e^{\frac{\alpha}{2}} t}\right|\left\|A^{\frac{1}{2}} \varphi\right\|_{H} \\
& +\left\|B^{\frac{1}{2}} s(t)\right\|_{H \rightarrow H}\left\|A^{1 / 2} B^{-\frac{1}{2}}\right\|_{H \rightarrow H}\left|e^{-\frac{\alpha}{2} t}\right|\left\|A^{\frac{1}{2}} \psi\right\|_{H} \\
& +\left\|A B^{-1}\right\|_{H \rightarrow H}\left[\|f(T)\|_{H}+\|c(t)\|_{H \rightarrow H}\|f(0)\|_{H}\right] \\
& +\left\|A B^{-1}\right\|_{H \rightarrow H} \int_{0}^{t}\|c(t-s)\|_{H \rightarrow H}\left\|f^{\prime}(s)\right\|_{H} d s \\
\leq & M_{3}(\delta)\left[\|A \varphi\|_{H}+\left\|A^{\frac{1}{2}} \psi\right\|_{H}+\|f(0)\|_{H}+\max _{0 \leq t \leq T}\left\|f^{\prime}(t)\right\|_{H}\right]
\end{aligned}
$$

for any $t \in[0, T]$. Then we get

$$
\max _{0 \leq t \leq T}\|A u(t)\|_{H} \leq M_{3}(\delta)\left[\|A \varphi\|_{H}+\left\|A^{\frac{1}{2}} \psi\right\|_{H}+\|f(0)\|_{H}+\max _{0 \leq t \leq T}\left\|f^{\prime}(t)\right\|_{H}\right]
$$

The estimate for $\max _{0 \leq t \leq T}\left\|\frac{d^{2} u}{d t^{2}}\right\|_{H}$ follows from the last estimate and the triangle inequality. Theorem 2.1. is proved.

Remark 2.1 All statements of Theorem 2.1 hold in an arbitrary Banach space $E$ under the assumptions (see, e.g., [24, 25]):

$$
\left\{\begin{array}{l}
\|c(t)\|_{E \rightarrow E} \leq M, \quad\left\|B^{\frac{1}{2}} s(t)\right\|_{E \rightarrow E} \leq M, \\
\left\|B^{-1 / 2} \varphi\right\|_{E} \leq M(\delta)\|\varphi\|_{E}, \quad\left\|A^{1 / 2} B^{-\frac{1}{2}}\right\|_{E \rightarrow E} \leq M(\delta) .
\end{array}\right.
$$

Now, we consider the application of this abstract theorem, Theorem 2.1. First, we consider the initial-value problem for the telegraph equations

$$
\left\{\begin{array}{l}
u_{t t}(t, x)+\alpha u_{t}(t, x)-\left(a(x) u_{x}\right)_{x}+\delta u(t, x)+\beta u(t, x) \\
=f(t, x), \quad 0<t<T, 0<x<l, \\
u(0, x)=\varphi(x), \quad u_{t}(0, x)=\psi(x), \quad 0 \leq x \leq l, \\
u(t, 0)=u(t, l), \quad u_{x}(t, 0)=u_{x}(t, l), \quad 0 \leq t \leq T .
\end{array}\right.
$$

Problem (10) has a unique smooth solution $u(t, x)$ for smooth $a(x) \geq a>0, x \in(0,1), \delta>0$, $a(l)=a(0), \varphi(x), \psi(x)(x \in[0, l])$, and $f(t, x)(t \in(0, T), x \in(0, l))$ functions. This allows 
us to reduce the problem (10) to the initial value (1) in a Hilbert space $H=L_{2}[0, l]$ with a self-adjoint positive definite operator $A^{x}$ defined by (10). Let us give a number corollaries of the abstract Theorem 2.1 .

Theorem 2.2 For solutions of the problem (10) the stability inequalities

$$
\begin{aligned}
& \max _{0 \leq t \leq T}\|u(t, \cdot)\|_{W_{2}^{1}[0, l]} \leq M_{1}\left[\max _{0 \leq t \leq T}\|f(t, \cdot)\|_{L_{2}[0, l]}+\|\varphi\|_{W_{2}^{1}[0, l]}+\|\psi\|_{L_{2}[0, l]}\right], \\
& \max _{0 \leq t \leq T}\|u(t, \cdot)\|_{W_{2}^{2}[0, l]}+\max _{0 \leq t \leq T}\left\|u_{t t}(t, \cdot)\right\|_{L_{2}[0, l]} \\
& \quad \leq M_{1}\left[\max _{0 \leq t \leq T}\left\|f_{t}(t, \cdot)\right\|_{L_{2}[0, l]}+\|f(0, \cdot)\|_{L_{2}[0, l]}+\|\varphi\|_{W_{2}^{2}[0, l]}+\|\psi\|_{W_{2}^{1}[0, l]}\right]
\end{aligned}
$$

hold, where $M_{1}$ does not depend on $f(t, x)$ and $\varphi(x), \psi(x)$.

Proof Problem (10) can be written in abstract form

$$
\left\{\begin{array}{l}
\frac{d^{2} u(t)}{d t^{2}}+\alpha \frac{d u(t)}{d t}+A u(t)+\beta u(t)=f(t) \quad(0 \leq t \leq T) \\
u(0)=\varphi, \quad u^{\prime}(0)=\psi
\end{array}\right.
$$

in a Hilbert space $L_{2}[0, l]$ of all square integrable functions defined on $[0, l]$ with selfadjoint positive definite operator $A=A^{x}$ by the formula

$$
A^{x} u(x)=-\left(a(x) u_{x}\right)_{x}+\sigma u(x)
$$

with the domain

$$
D\left(A^{x}\right)=\left\{u(x): u, u_{x},\left(a(x) u_{x}\right)_{x} \in L_{2}[0, l], u(0)=u(l), u^{\prime}(0)=u^{\prime}(l)\right\}
$$

Here, $f(t)=f(t, x)$ and $u(t)=u(t, x)$ are known and unknown abstract functions defined on $[0, l]$ with the values in $H=L_{2}[0, l]$. Therefore, estimates (11) and (12) follow from estimates (5), (6), and (7). Thus, Theorem 2.2 is proved.

Second, let $\Omega \subset R^{n}$ be a bounded open domain with smooth boundary $S, \bar{\Omega}=\Omega \cup S$. In $[0, T] \times \Omega$ we consider the boundary value problem for telegraph equations

$$
\left\{\begin{array}{l}
u_{t t}(t, x)+\alpha u_{t}(t, x)-\sum_{r=1}^{n}\left(a_{r}(x) u_{x^{r}}\right) x_{r}+\beta u(t, x) \\
\quad=f(t, x), \quad x=\left(x_{1}, \ldots, x_{n}\right) \in \Omega, 0<t<T, \\
u(0, x)=\varphi(x), \quad \frac{\partial u(0, x)}{\partial t}=\psi(x), \quad x \in \bar{\Omega} \\
u(t, x)=0, \quad x \in S, 0 \leq t \leq T,
\end{array}\right.
$$

where $a_{r}(x)(x \in \Omega), \varphi(x), \psi(x)(x \in \bar{\Omega})$ and $f(t, x), t \in(0, T), x \in \Omega$, are given smooth functions and $a_{r}(x)>0$. We introduce the Hilbert space $L_{2}(\bar{\Omega})$, the space of all integrable functions defined on $\bar{\Omega}$, equipped with the norm

$$
\|f\|_{L_{2}(\bar{\Omega})}=\left\{\int \cdots \int_{x \in \bar{\Omega}}|f(x)|^{2} d x_{1} \cdots d x_{n}\right\}^{\frac{1}{2}} .
$$


Theorem 2.3 For solutions of the problem (15) the stability inequalities

$$
\begin{aligned}
& \max _{0 \leq t \leq T}\|u(t, \cdot)\|_{W_{2}^{1}(\bar{\Omega})} \leq M\left[\max _{0 \leq t \leq T}\|f(t, \cdot)\|_{L_{2}(\bar{\Omega})}+\|\varphi\|_{W_{2}^{1}(\bar{\Omega})}+\|\psi\|_{L_{2}(\bar{\Omega})}\right], \\
& \max _{0 \leq t \leq T}\|u(t, \cdot)\|_{W_{2}^{2}(\bar{\Omega})}+\max _{0 \leq t \leq T}\left\|u_{t t}(t, \cdot)\right\|_{L_{2}(\bar{\Omega})} \\
& \quad \leq M_{1}\left[\max _{0 \leq t \leq T}\left\|f_{t}(t, \cdot)\right\|_{L_{2}(\bar{\Omega})}+\|f(0, \cdot)\|_{L_{2}(\bar{\Omega})}+\|\varphi\|_{W_{2}^{2}(\bar{\Omega})}+\|\psi\|_{W_{2}^{1}(\bar{\Omega})}\right]
\end{aligned}
$$

hold, where $M_{1}$ does not depend on $f(t, x)$ and $\varphi(x), \psi(x)$.

Proof Problem (15) can be written in the abstract form (13) in Hilbert space $L_{2}(\bar{\Omega})$ with self-adjoint positive definite operator $A=A^{x}$ defined by formula

$$
A^{x} u(x)=-\sum_{r=1}^{n}\left(a_{r}(x) u_{x_{r}}\right)_{x_{r}}+\sigma u(x)
$$

with domain

$$
D\left(A^{x}\right)=\left\{u(x): u(x), u_{x_{r}}(x),\left(a_{r}(x) u_{x_{r}}\right)_{x_{r}} \in L_{2}(\bar{\Omega}), 1 \leq r \leq n, u(x)=0, x \in S\right\} .
$$

Here, $f(t)=f(t, x)$ and $u(t)=u(t, x)$ are known and unknown abstract functions defined on $\bar{\Omega}$ with the values in $H=L_{2}(\bar{\Omega})$. So, estimates (16) and (17) follow from estimates (5), (6), and (7) and the following theorem.

Theorem 2.4 For the solutions of the elliptic differential problem [26]

$$
\left\{\begin{array}{l}
A^{x} u(x)=\omega(x), \quad x \in \Omega \\
u(x)=0, \quad x \in S
\end{array}\right.
$$

the following coercivity inequality holds:

$$
\sum_{r=1}^{n}\left\|u_{x_{r} x_{r}}\right\|_{L_{2}(\bar{\Omega})} \leq M_{1}\|\omega\|_{L_{2}(\bar{\Omega})} .
$$

Here $M_{1}$ does not depend on $\omega(x)$.

In Section 3, the difference schemes of the first and second order of accuracy for the approximate solution of problem (1) are investigated. Stability estimates for the solution of these difference schemes are established. In applications, difference schemes for the approximate solution of the two mixed problems (10) and (15) are presented. Stability estimates for the solution of these difference schemes are established.

\section{Stability of two-step difference schemes}

First, we consider the approximation of first order in $t$ of the two-step difference scheme for the numerical solution of the initial value problem (1)

$$
\left\{\begin{array}{l}
\frac{u_{k+1}-2 u_{k}+u_{k-1}}{\tau^{2}}+\alpha \frac{u_{k+1}-u_{k-1}}{2 \tau}+A u_{k+1}+\beta u_{k+1}=f_{k} \\
f_{k}=f\left(t_{k+1}\right), 1 \leq k \leq N-1, N \tau=T \\
u_{0}=\varphi, \quad \frac{u_{1}-u_{0}}{\tau}+\left(A+\left(\beta-\frac{\alpha^{2}}{4}\right) I\right) \tau u_{1}=\frac{1}{1+\frac{\alpha}{2} \tau} \psi
\end{array}\right.
$$


Now, we will consider operators $R, \widetilde{R}$ defined by

$$
\begin{aligned}
& R=\left(I+i \sqrt{\left.\left(1-\frac{\alpha \tau}{2}\right)\left(\left(1+\frac{\alpha \tau}{2}\right) I+\tau^{2}\left(B+\frac{\alpha^{2}}{4} I\right)\right)-I\right)}\right) \\
& \widetilde{R}=\left(I-i \sqrt{\left.\left(1-\frac{\alpha \tau}{2}\right)\left(\left(1+\frac{\alpha \tau}{2}\right) I+\tau^{2}\left(B+\frac{\alpha^{2}}{4} I\right)\right)-I\right)}\right) \\
& P=\left(\left(1+\frac{\alpha \tau}{2}\right) I+\tau^{2}\left(B+\frac{\alpha^{2}}{4} I\right)\right)^{-1}
\end{aligned}
$$

and we will introduce the following operators:

$$
\begin{aligned}
R_{1}= & \left(\left(\alpha \tau+\frac{3 \tau^{2} B}{2}+\frac{3 \alpha^{2} \tau^{2}}{8}\right)\right. \\
& \left.+i \tau B^{1 / 2}\left(2+\alpha \tau+\tau^{2} B+\frac{\alpha^{2} \tau^{2}}{4}\right) \sqrt{4-2 \alpha \tau-\frac{\alpha^{3} \tau}{2} B^{-1}}\right) \\
& \times\left(\left(-2 i \tau B^{1 / 2} \sqrt{4-2 \alpha \tau-\frac{\alpha^{3} \tau}{2} B^{-1}}\right)\left(2+\alpha \tau+\tau^{2} B+\frac{\alpha^{2} \tau^{2}}{4}\right)\right)^{-1}, \\
R_{2}= & \left(\tau \sqrt{4+B+\frac{\alpha^{2} \tau^{2}}{2}+\frac{\alpha^{4} \tau^{2}}{16} B^{-1}}\right)\left(2+\alpha \tau+2 \tau^{2} B+\frac{\alpha^{2} \tau^{2}}{2}\right) \\
& \times\left(-i B^{\frac{1}{2}}\left(2+\alpha \tau+\tau^{2} B+\frac{\alpha^{2} \tau^{2}}{4}\right) \sqrt{4-2 \alpha \tau-\frac{\alpha^{3} \tau}{2} B^{-1}}\right)^{-1}, \\
R_{3}= & \left(\tau B^{\frac{1}{2}}\left(2+\alpha \tau+2 \tau^{2} B+\frac{\alpha^{2} \tau^{2}}{2}\right)\right) \\
& \times\left(\left(2+\alpha \tau+\tau^{2} B+\frac{\alpha^{2} \tau^{2}}{4}\right)\left(i \tau B^{\frac{1}{2}} \sqrt{4-2 \alpha \tau-\frac{\alpha^{3} \tau}{2} B^{-1}}\right)\right)^{-1}, \\
R_{4}= & \left(4+4 \tau^{2} B-2 \alpha \tau^{3} B-\frac{\alpha^{3} \tau^{3}}{2}\right) \\
R_{6}= & \left(2-i \tau B^{\frac{1}{2}} \sqrt{4-2 \alpha \tau-\frac{\alpha^{3} \tau}{2} B^{-1}}\right) \\
& \times\left(\left(-i B^{\frac{1}{2}} \sqrt{4-2 \alpha \tau-\frac{\alpha^{3} \tau}{2} B^{-1}}\right)\right. \\
& \left.\times\left(2+\alpha \tau+2 \tau^{2} B+\frac{\alpha^{2} \tau^{2}}{2}\right)(2-\alpha \tau)\right)^{-1}, \\
R_{5}= & \left(-\alpha \tau-2 \tau^{2} B-\frac{\alpha^{2} \tau^{2}}{2}+i \tau B^{\frac{1}{2}} \sqrt{4-2 \alpha \tau-\frac{\alpha^{3} \tau}{2} B^{-1}}\right) \\
& \left(\left(2+\alpha \tau+2 \tau^{2} B+\frac{\alpha^{2} \tau^{2}}{2}\right)\right)^{-1} \\
& \left(\alpha \tau^{2} B+\frac{\alpha^{2} \tau^{2}}{2}-i \tau B^{\frac{1}{2}} \sqrt{4-2 \alpha \tau-\frac{\alpha^{3} \tau}{2} B^{-1}}\right)^{-1} \\
& (2-2)
\end{aligned}
$$

and their conjugates $\widetilde{R}_{1}, \widetilde{R}_{5}, \widetilde{R}_{6}$. Let us give one lemma, which will be needed below. 


\section{Lemma 3.1 The following estimates hold:}

$$
\left\{\begin{array}{l}
\|R\|_{H \rightarrow H} \leq 1, \quad\|\widetilde{R}\|_{H \rightarrow H} \leq 1, \\
\left\|R_{1}\right\|_{H \rightarrow H} \leq 1, \quad\left\|\widetilde{R}_{1}\right\|_{H \rightarrow H} \leq 1, \\
\left\|B^{1 / 2} R_{2}\right\|_{H \rightarrow H} \leq 1, \quad\left\|\tau B^{1 / 2} R_{3}\right\|_{H \rightarrow H} \leq 1, \\
\left\|B^{1 / 2} R_{4}\right\|_{H \rightarrow H} \leq 1, \quad\left\|B^{-1 / 2} R_{5}\right\|_{H \rightarrow H} \leq \tau, \\
\left\|B^{-1 / 2} \widetilde{R}_{5}\right\|_{H \rightarrow H} \leq \tau, \quad \\
\left\|\tau B^{1 / 2} R_{6}\right\|_{H \rightarrow H} \leq 1, \quad\left\|\tau B^{1 / 2} \widetilde{R}_{6}\right\|_{H \rightarrow H} \leq 1 .
\end{array}\right.
$$

Theorem 3.1 Suppose that the assumption (2) holds and $\varphi \in D(A), \psi \in D\left(A^{\frac{1}{2}}\right)$. Then for the solution of difference scheme (19) the stability estimates

$$
\begin{gathered}
\max _{1 \leq k \leq N}\left\|u_{k}\right\|_{H} \leq M\left\{\max _{1 \leq k \leq N-1}\left\|A^{-1 / 2} f_{k}\right\|_{H}+\left\|A^{-1 / 2} \psi\right\|_{H}+\|\varphi\|_{H}\right\}, \\
\max _{1 \leq k \leq N}\left\|A^{1 / 2} u_{k}\right\|_{H} \leq M\left\{\max _{1 \leq k \leq N-1}\left\|f_{k}\right\|_{H}+\|\psi\|_{H}+\left\|A^{1 / 2} \varphi\right\|_{H}\right\}, \\
\max _{1 \leq k \leq N}\left\|A u_{k}\right\|_{H} \leq M\left\{\max _{2 \leq k \leq N-1}\left\|\frac{1}{\tau}\left(f_{k}-f_{k-1}\right)\right\|_{H}\right. \\
\left.+\left\|f_{1}\right\|_{H}+\left\|A^{1 / 2} \psi\right\|_{H}+\|A \varphi\|_{H}\right\}
\end{gathered}
$$

hold, where $M$ does not depend on $\tau, \varphi, \psi$, and $f_{k}, 1 \leq s \leq N-1$.

Proof We will obtain the formula for the solution of the problem (1). We can rewrite (1) into the following difference problem:

$$
\left\{\begin{array}{c}
\left(1-\frac{\alpha \tau}{2}\right) I u_{k-1}-2 I u_{k}+\left(\left(1+\frac{\alpha \tau}{2}\right) I+\tau^{2}\left(B+\frac{\alpha^{2}}{4} I\right)\right) u_{k+1} \\
\quad=\tau^{2} f_{k}, \quad 1 \leq k \leq N-1, \\
u_{0}=\varphi, \quad u_{1}=\left(I+B \tau^{2}\right)^{-1} \varphi+\tau\left(1+\frac{\alpha \tau}{2}\right)^{-1}\left(I+B \tau^{2}\right)^{-1} \psi
\end{array}\right.
$$

It is clear that there exists a unique solution of this initial-value problem and for the solution of (25), the following formula is satisfied (see [3]):

$$
\begin{aligned}
u_{0}= & \varphi, \quad u_{1}=\left(I+B \tau^{2}\right)^{-1} \varphi+\tau\left(1+\frac{\alpha \tau}{2}\right)^{-1}\left(I+B \tau^{2}\right)^{-1} \psi \\
u_{k}= & R \widetilde{R}(\widetilde{R}-R)^{-1}\left[R^{k-1}-\widetilde{R}^{k-1}\right] \varphi \\
& +(\widetilde{R}-R)^{-1}\left(\widetilde{R}^{k}-R^{k}\right)\left[\left(I+B \tau^{2}\right)^{-1} \varphi+\tau\left(1+\frac{\alpha \tau}{2}\right)^{-1}\left(I+B \tau^{2}\right)^{-1} \psi\right] \\
& +\sum_{s=1}^{k-1} R \widetilde{R}\left((\widetilde{R}-R)\left(1-\frac{\alpha \tau}{2}\right)\right)^{-1}\left[\widetilde{R}^{k-s}-R^{k-s}\right] \tau^{2} f_{s} .
\end{aligned}
$$

Using the spectral property of the self-adjoint positive definite operators, we get

$$
\begin{aligned}
& \left\|\left(I+B \tau^{2}\right)^{-1}\right\|_{H \rightarrow H} \leq 1 \\
& \left\|\tau B^{\frac{1}{2}}\left(I+B \tau^{2}\right)^{-1}\right\|_{H \rightarrow H} \leq 1, \quad\left\|\tau^{2} B\left(I+B \tau^{2}\right)^{-1}\right\|_{H \rightarrow H} \leq 1 .
\end{aligned}
$$


Then, using the triangle inequality, we get

$$
\begin{aligned}
\left\|u_{1}\right\|_{H} \leq & \left\|\left(I+B \tau^{2}\right)^{-1}\right\|_{H \rightarrow H}\|\varphi\|_{H} \\
& +\left(1+\frac{\alpha \tau}{2}\right)^{-1}\left\|A^{\frac{1}{2}} B^{-\frac{1}{2}}\right\|_{H \rightarrow H}\left\|\tau B^{\frac{1}{2}}\left(I+B \tau^{2}\right)^{-1}\right\|_{H \rightarrow H}\left\|A^{-1 / 2} \psi\right\|_{H} \\
\leq & \left\|A^{-1 / 2} \psi\right\|_{H}+\|\varphi\|_{H} .
\end{aligned}
$$

In exactly the same manner, one establishes

$$
\begin{aligned}
\left\|A^{\frac{1}{2}} u_{1}\right\|_{H} \leq & \left\|\left(I+B \tau^{2}\right)^{-1}\right\|_{H \rightarrow H}\left\|A^{\frac{1}{2}} \varphi\right\|_{H} \\
& +\left(1+\frac{\alpha \tau}{2}\right)^{-1}\left\|A^{\frac{1}{2}} B^{-\frac{1}{2}}\right\|_{H \rightarrow H}\left\|\tau B^{\frac{1}{2}}\left(I+B \tau^{2}\right)^{-1}\right\|_{H \rightarrow H}\|\psi\|_{H} \\
\leq & \|\psi\|_{H}+\left\|A^{\frac{1}{2}} \varphi\right\|_{H^{\prime}} \\
\left\|A u_{1}\right\|_{H} \leq & \left\|\left(I+B \tau^{2}\right)^{-1}\right\|_{H \rightarrow H}\|A \varphi\|_{H} \\
& +\left(1+\frac{\alpha \tau}{2}\right)^{-1}\left\|A^{\frac{1}{2}} B^{-\frac{1}{2}}\right\|_{H \rightarrow H}\left\|\tau B^{\frac{1}{2}}\left(I+B \tau^{2}\right)^{-1}\right\|_{H \rightarrow H}\|\psi\|_{H} \\
\leq & \left\|A^{\frac{1}{2}} \psi\right\|_{H}+\|A \varphi\|_{H} .
\end{aligned}
$$

Using the spectral property of the self-adjoint positive definite operators, we get

$$
\begin{array}{lll}
\|R\|_{H \rightarrow H} \leq 1, & \left\|\tau B^{\frac{1}{2}} R\right\|_{H \rightarrow H} \leq 1, & \left\|\tau^{2} B R\right\|_{H \rightarrow H} \leq 1, \\
\|\widetilde{R}\|_{H \rightarrow H} \leq 1, & \left\|\tau B^{\frac{1}{2}} \widetilde{R}\right\|_{H \rightarrow H} \leq 1, & \left\|\tau^{2} B \widetilde{R}\right\|_{H \rightarrow H} \leq 1 .
\end{array}
$$

Now, we will establish estimates for $\left\|u_{k}\right\|_{H}, k \geq 2$. Using (26), the estimates for (28), (31), (32), (21), and the triangle inequality, we get

$$
\begin{aligned}
\left\|u_{k}\right\|_{H} \leq & \frac{1}{2}\left(\left\|\widetilde{R}_{1}\right\|_{H \rightarrow H}\left\|R^{k}\right\|_{H \rightarrow H}+\left\|R_{1}\right\|_{H \rightarrow H}\left\|\widetilde{R}^{k}\right\|_{H \rightarrow H}\right)\|\varphi\|_{H} \\
& +\frac{1}{2}\left(\left\|A^{1 / 2} R_{2}\right\|_{H \rightarrow H}\left\|\widetilde{R}^{k}\right\|_{H \rightarrow H}+\left\|A^{1 / 2} R_{2}\right\|_{H \rightarrow H}\left\|R^{k}\right\|_{H \rightarrow H}\right) \\
& \times\left\|\left(I+B \tau^{2}\right)^{-1}\right\|_{H \rightarrow H}\left[\left\|A^{-1 / 2}\right\|_{H \rightarrow H}\|\varphi\|_{H}+\tau\left(1+\frac{\alpha \tau}{2}\right)^{-1}\left\|A^{-1 / 2} \psi\right\|_{H}\right] \\
& +\frac{1}{2}\left\|\tau A^{1 / 2} R_{4}\right\|_{H \rightarrow H} \sum_{s=1}^{N-1}\left[\left\|\widetilde{R}^{k-s}\right\|_{H \rightarrow H}+\left\|R^{k-s}\right\|_{H \rightarrow H}\right] \tau\left\|A^{-1 / 2} f_{s}\right\|_{H} \\
\leq & M\left\{\sum_{s=1}^{N-1}\left\|A^{-1 / 2} f_{s}\right\|_{H} \tau+\|\varphi\|_{H}+\left\|A^{-1 / 2} \psi\right\|_{H}\right\}
\end{aligned}
$$

for any $k \geq 2$. Combining the estimates for $\left\|u_{k}\right\|$ for any $k$, we obtain (22).

Applying $A^{1 / 2}$ to (26) and using estimates for (28), (31), (32), (21), and the triangle inequality, we get

$$
\begin{aligned}
\left\|A^{1 / 2} u_{k}\right\|_{H} \leq & \frac{1}{2}\left(\left\|\widetilde{R}_{1}\right\|_{H \rightarrow H}\left\|R^{k}\right\|_{H \rightarrow H}+\left\|R_{1}\right\|_{H \rightarrow H}\left\|\widetilde{R}^{k}\right\|_{H \rightarrow H}\right)\left\|A^{1 / 2} \varphi\right\|_{H} \\
& +\frac{1}{2}\left(\left\|A^{1 / 2} R_{2}\right\|_{H \rightarrow H}\left\|\widetilde{R}^{k}\right\|_{H \rightarrow H}+\left\|A^{1 / 2} R_{2}\right\|_{H \rightarrow H}\left\|R^{k}\right\|_{H \rightarrow H}\right)
\end{aligned}
$$




$$
\begin{aligned}
\times & \left\|\left(I+B \tau^{2}\right)^{-1}\right\|_{H \rightarrow H}\left[\left\|A^{-1 / 2}\right\|_{H \rightarrow H}\left\|A^{1 / 2} \varphi\right\|_{H}+\tau\left(1+\frac{\alpha \tau}{2}\right)^{-1}\|\psi\|_{H}\right] \\
+ & \frac{1}{2}\left\|\tau A^{1 / 2} R_{4}\right\|_{H \rightarrow H} \sum_{s=1}^{N-1}\left[\left\|\widetilde{R}^{k-s}\right\|_{H \rightarrow H}+\left\|R^{k-s}\right\|_{H \rightarrow H}\right] \tau\left\|f_{s}\right\|_{H} \\
\leq & M\left\{\sum_{s=1}^{N-1}\left\|f_{s}\right\|_{H} \tau+\left\|A^{1 / 2} \varphi\right\|_{H}+\|\psi\|_{H}\right\}
\end{aligned}
$$

for any $k \geq 2$. Combining the estimates for $\left\|A^{1 / 2} u_{k}\right\|$ for any $k$, we obtain (23). Finally, applying Abel's formula to (26), we can write

$$
\begin{aligned}
u_{k}= & \frac{1}{2}\left[\widetilde{R}_{1} R^{k}-R_{1} \widetilde{R}^{k}\right] \varphi+\frac{1}{2}\left[\widetilde{R}^{k}-R^{k}\right] R_{2}\left[\left(I+B \tau^{2}\right)^{-1} \varphi+\tau\left(1+\frac{\alpha \tau}{2}\right)^{-1}\left(I+B \tau^{2}\right)^{-1} \psi\right] \\
& +\frac{1}{2}\left[\widetilde{R}^{k}-R^{k}\right] R_{3} \tau^{2} f_{1}+\frac{1}{2} \tau^{2} R_{4}\left(\sum_{s=2}^{k-1}\left[R_{6} \widetilde{R}^{k-s}-\widetilde{R}_{6} R^{k-s}\right]\left(f_{s}-f_{s-1}\right)\right. \\
& \left.+\left(\widetilde{R}_{6}-R_{6}\right) f_{k-1}-\left[\widetilde{R}_{6} \widetilde{R}^{k-1}-R_{6} R^{k-1}\right] f_{1}\right), \quad 2 \leq k \leq N
\end{aligned}
$$

Next, applying $A$ to (33) and using estimates for (28), (31), (32), (21), we get

$$
\begin{aligned}
\left\|A u_{k}\right\|_{H} \leq & \frac{1}{2}\left(\left\|\widetilde{R}_{1}\right\|_{H \rightarrow H}\left\|R^{k}\right\|_{H \rightarrow H}+\left\|R_{1}\right\|_{H \rightarrow H}\left\|\widetilde{R}^{k}\right\|_{H \rightarrow H}\right)\|A \varphi\|_{H} \\
& +\frac{1}{2}\left(\left\|A^{1 / 2} R_{2}\right\|_{H \rightarrow H}\left\|\widetilde{R}^{k}\right\|_{H \rightarrow H}+\left\|A^{1 / 2} R_{2}\right\|_{H \rightarrow H}\left\|R^{k}\right\|_{H \rightarrow H}\right) \\
& \times\left\|\left(I+B \tau^{2}\right)^{-1}\right\|_{H \rightarrow H}\left[\left\|A^{-1 / 2}\right\|_{H \rightarrow H}\|A \varphi\|_{H}+\tau\left(1+\frac{\alpha \tau}{2}\right)^{-1}\left\|A^{1 / 2} \psi\right\|_{H}\right] \\
& +\frac{1}{2}\left(\left\|\tau A^{1 / 2} R_{3}\right\|_{H \rightarrow H}\left\|\widetilde{R}^{k}\right\|_{H \rightarrow H}+\left\|\tau A^{1 / 2} R_{3}\right\|_{H \rightarrow H}\left\|R^{k}\right\|_{H \rightarrow H}\right) \tau\left\|A^{1 / 2} f_{1}\right\|_{H} \\
& +\frac{1}{2}\left\|\tau A^{1 / 2} R_{4}\right\|_{H \rightarrow H} \sum_{s=2}^{N-1}\left[\left\|\tau A^{1 / 2} R_{6}\right\|_{H \rightarrow H}\left\|R^{k-s}\right\|_{H \rightarrow H}\right. \\
& \left.+\left\|\tau A^{1 / 2} \widetilde{R}_{6}\right\|_{H \rightarrow H}\left\|\widetilde{R}^{k-s}\right\|_{H \rightarrow H}\right] \\
& \times\left\|f_{s}-f_{s-1}\right\|_{H}+\left[\left\|\tau A^{1 / 2} \widetilde{R}_{6}\right\|_{H \rightarrow H}+\left\|\tau A^{1 / 2} R_{6}\right\|_{H \rightarrow H}\right]\left\|f_{k-1}\right\|_{H} \\
& +\left[\left\|\tau A^{1 / 2} \widetilde{R}_{6}\right\|_{H \rightarrow H}\left\|\widetilde{R}^{k-1}\right\|_{H \rightarrow H}+\left\|\tau A^{1 / 2} R_{6}\right\|_{H \rightarrow H}\left\|R^{k-1}\right\|_{H \rightarrow H}\right]\left\|f_{1}\right\|_{H} \\
\leq & M\left\{\sum_{s=2}^{N-1}\left\|f_{s}-f_{s-1}\right\|_{H}+\left\|f_{1}\right\|_{H}+\|A \varphi\|_{H}+\left\|A^{1 / 2} \psi\right\|_{H}\right\}
\end{aligned}
$$

for any $k \geq 2$. Combining the estimates for $\left\|A u_{k}\right\|_{H}$ for any $k$, we obtain (24). Theorem 3.1 is proved.

Second, we consider two types of approximations of second order in $t$ by two-step difference schemes for the numerical solution of the initial value problem (1):

$$
\left\{\begin{array}{l}
\frac{u_{k+1}-2 u_{k}+u_{k-1}}{\tau^{2}}+\alpha \frac{u_{k+1}-u_{k-1}}{2 \tau}+\frac{A}{2}\left(u_{k+1}+u_{k-1}\right) \\
\quad+\frac{\beta}{2}\left(u_{k+1}+u_{k-1}\right)=f_{k}, \quad f_{k}=f\left(t_{k}\right), 1 \leq k \leq N-1, \\
u_{0}=\varphi \\
\frac{u_{1}-u_{0}}{\tau}+\frac{\tau}{4} B u_{1}+\frac{1}{1+\frac{\alpha}{4} \tau}\left(\frac{1}{4} B-\frac{\alpha \tau B}{16}+\frac{\alpha^{2}}{8} I\right) \tau u_{0}=\frac{1-\frac{\alpha}{4} \tau}{1+\frac{\alpha}{4} \tau}\left(\psi+\frac{\tau}{2} f_{0}\right), \quad f_{0}=f(0),
\end{array}\right.
$$




$$
\left\{\begin{array}{l}
\frac{u_{k+1}-2 u_{k}+u_{k-1}}{\tau^{2}}+\alpha \frac{u_{k+1}-u_{k-1}}{2 \tau}+\frac{A}{2} u_{k}+\frac{A}{4}\left(u_{k+1}+u_{k-1}\right) \\
\quad+\frac{\beta}{2} u_{k}+\frac{\beta}{4}\left(u_{k+1}+u_{k-1}\right)=f_{k}, \quad f_{k}=f\left(t_{k}\right), 1 \leq k \leq N-1, \\
u_{0}=\varphi \\
\frac{u_{1}-u_{0}}{\tau}+\frac{\tau}{4} B u_{1}+\frac{1}{1+\frac{\alpha}{4} \tau}\left(\frac{1}{4} B-\frac{\alpha \tau B}{16}+\frac{\alpha^{2}}{8} I\right) \tau u_{0}=\frac{1-\frac{\alpha}{4} \tau}{1+\frac{\alpha}{4} \tau}\left(\psi+\frac{\tau}{2} f_{0}\right), \quad f_{0}=f(0) .
\end{array}\right.
$$

Theorem 3.2 Suppose that the assumption (2) holds and $\varphi \in D(A), \psi \in D\left(A^{\frac{1}{2}}\right)$. Then for the solution of difference schemes (34) and (35) the following stability estimates hold:

$$
\begin{gathered}
\max _{1 \leq k \leq N}\left\|u_{k}\right\|_{H} \leq M\left\{\max _{0 \leq k \leq N-1}\left\|A^{-1 / 2} f_{k}\right\|_{H}+\left\|A^{-1 / 2} \psi\right\|_{H}+\|\varphi\|_{H}\right\} \\
\max _{1 \leq k \leq N}\left\|A^{1 / 2} u_{k}\right\|_{H} \leq M\left\{\max _{0 \leq k \leq N-1}\left\|f_{k}\right\|_{H}+\|\psi\|_{H}+\left\|A^{1 / 2} \varphi\right\|_{H}\right\} \\
\max _{1 \leq k \leq N}\left\|A u_{k}\right\|_{H} \leq M\left\{\max _{1 \leq k \leq N-1}\left\|\frac{1}{\tau}\left(f_{k}-f_{k-1}\right)\right\|_{H}\right. \\
\left.+\left\|f_{0}\right\|_{H}+\left\|A^{1 / 2} \psi\right\|_{H}+\|A \varphi\|_{H}\right\}
\end{gathered}
$$

hold, where $M$ does not depend on $\tau, \varphi, \psi$, and $f_{k}, 0 \leq s \leq N-1$.

The proof of Theorem 3.2 is based on the formulas for the solution of the difference schemes (34) and (35), on the estimates for the step operators, and on the self-adjointness and positivity of operator $A$.

Now, we consider applications of the main theorem, Theorem 3.1. First, we consider the boundary value problem (10). The discretization of problem (10) is carried out in two steps. In the first step, we define the grid space

$$
[0, l]_{h}=\left\{x=x_{n}: x_{n}=n h, 0 \leq n \leq M, M h=l\right\}
$$

Let us introduce the Hilbert space $L_{2 h}=L_{2}\left([0, l]_{h}\right)$ of the grid functions $\varphi^{h}(x)=\left\{\varphi_{n}\right\}_{0}^{M}$ defined on $[0, l]_{h}$, equipped with the norm

$$
\left\|\varphi^{h}\right\|_{L_{2 h}}=\left(\sum_{x \in[0, L]_{h}}|\varphi(x)|^{2} h\right)^{1 / 2} .
$$

To the differential operator $A^{x}$ defined by the formula (14), we assign the difference operator $A_{h}^{x}$ by the formula

$$
A_{h}^{x} \varphi^{h}(x)=\left\{-\left(a(x) \varphi_{\bar{x}}\right)_{x, n}+\delta \varphi_{n}\right\}_{1}^{M-1}
$$

acting in the space of grid functions $\varphi^{h}(x)=\left\{\varphi_{n}\right\}_{0}^{M}$ satisfying the conditions $\varphi_{0}=\varphi_{M}, \varphi_{1}-$ $\varphi_{0}=\varphi_{M}-\varphi_{M-1}$. It is well known that $A_{h}^{x}$ is a self-adjoint positive definite operator in $L_{2 h}$. With the help of $A_{h}^{x}$, we reach the boundary value problem

$$
\left\{\begin{array}{l}
u_{t t}^{h}(t, x)+\alpha u_{t}^{h}(t, x)+A_{h}^{x} u^{h}(t, x)+\beta u^{h}(t, x) \\
\quad=f^{h}(t, x), \quad 0<t<T, x \in[0, l]_{h}, \\
u^{h}(0, x)=\varphi^{h}(x), \quad u_{t}^{h}(0, x)=\psi^{h}(x), \quad x \in[0, l]_{h} .
\end{array}\right.
$$


In the second step, we replace (37) with the difference scheme (19),

$$
\left\{\begin{array}{l}
\frac{u_{k+1}^{h}(x)-2 u_{k}^{h}(x)+u_{k-1}^{h}(x)}{\tau^{2}}+\alpha \frac{u_{k+1}^{h}(x)-u_{k-1}^{h}(x)}{2 \tau}+A_{h}^{x} u_{k+1}^{h}(x)+\beta u_{k+1}^{h}(x)=f_{k}^{h}(x), \\
f_{k}^{h}(x)=f^{h}\left(t_{k+1}, x\right), t_{k}=k \tau, 1 \leq k \leq N-1, x \in[0, l]_{h}, N \tau=T, \\
u_{0}^{h}(x)=\varphi^{h}(x), \\
\frac{u_{1}^{h}(x)-u_{0}^{h}(x)}{\tau}+\left(A_{h}^{x}+\left(\beta-\frac{\alpha^{2}}{4}\right) I_{h}\right) \tau u_{1}^{h}(x)=\frac{1}{1+\frac{\alpha}{2} \tau} \psi^{h}(x), \quad x \in[0, l]_{h} .
\end{array}\right.
$$

Theorem 3.3 For the solution $\left\{u_{k}^{h}(x)\right\}_{0}^{N}$ of problem (38) the following stability estimates:

$$
\begin{aligned}
& \max _{1 \leq k \leq N}\left\|u_{k}^{h}\right\|_{L_{2 h}} \leq M_{1}\left\{\max _{1 \leq k \leq N-1}\left\|f_{k}^{h}\right\|_{L_{2 h}}+\left\|\psi^{h}\right\|_{L_{2 h}}+\left\|\varphi^{h}\right\|_{L_{2 h}}\right\} \\
& \max _{1 \leq k \leq N}\left\|u_{k}^{h}\right\|_{W_{2 h}^{1}} \leq M_{1}\left\{\max _{1 \leq k \leq N-1}\left\|f_{k}^{h}\right\|_{L_{2 h}}+\left\|\psi^{h}\right\|_{L_{2 h}}+\left\|\varphi^{h}\right\|_{W_{2 h}^{1}}\right\} \\
& \max _{1 \leq k \leq N}\left\|u_{k}^{h}\right\|_{W_{2 h}^{2} \leq} \leq M_{1}\left\{\max _{2 \leq k \leq N-1}\left\|\frac{1}{\tau}\left(f_{k}^{h}-f_{k-1}^{h}\right)\right\|_{L_{2 h}}\right. \\
&\left.+\left\|f_{1}^{h}\right\|_{L_{2 h}}+\left\|\psi^{h}\right\|_{W_{2 h}^{1}}+\left\|\varphi^{h}\right\|_{W_{2 h}^{2}}\right\}
\end{aligned}
$$

hold, where $M_{1}$ and $M_{2}$ do not depend on $\varphi^{h}(x), \psi^{h}(x)$ and $f_{k}^{h}(x), 1 \leq k \leq N-1$.

Proof Difference scheme (38) can be written in abstract form

$$
\left\{\begin{array}{l}
\frac{u_{k+1}^{h}-2 u_{k}^{h}+u_{k-1}^{h}}{\tau^{2}}+\alpha \frac{u_{k+1}^{h}-u_{k-1}^{h}}{2 \tau}+A_{h} u_{k+1}^{h}+\beta u_{k+1}^{h}=f_{k}^{h}, \\
1 \leq k \leq N-1, N \tau=T, \\
u_{0}^{h}=\varphi^{h}, \quad \frac{u_{1}^{h}-u_{0}^{h}}{\tau}+\left(A_{h}+\left(\beta-\frac{\alpha^{2}}{4}\right) I_{h}\right) \tau u_{1}^{h}=\frac{1}{1+\frac{\alpha}{2} \tau} \psi^{h}
\end{array}\right.
$$

in a Hilbert space $L_{2 h}$ with self-adjoint positive definite operator $A_{h}=A_{h}^{x}$ by formula (36). Here, $f_{k}^{h}=f_{k}^{h}(x)$ and $u_{k}^{h}=u_{k}^{h}(x)$ are known and unknown abstract mesh functions defined on $[0, l]_{h}$ with the values in $H=L_{2 h}$. Therefore, estimates of Theorem 3.3 follow from estimates (22), (23), and (24). Thus, Theorem 3.3 is proved.

Second, we consider the boundary value problem (15). The discretization of problem (15) is carried out in two steps. In the first step, we define the grid space

$$
\begin{aligned}
& \bar{\Omega}_{h}=\left\{x=x_{r}=\left(h_{1} j_{1}, \ldots, h_{n} j_{n}\right), j=\left(j_{1}, \ldots, j_{h}\right), 0 \leq j_{r} \leq N_{r}, N_{r} h_{r}=1, r=1, \ldots, n\right\}, \\
& \Omega_{h}=\bar{\Omega}_{h} \cap \Omega, \quad S_{h}=\bar{\Omega}_{h} \cap S,
\end{aligned}
$$

and introduce the Hilbert space $L_{2 h}=L_{2}\left(\bar{\Omega}_{h}\right)$ of the grid functions $\varphi^{h}(x)=\left\{\varphi\left(h_{1} j_{1}, \ldots\right.\right.$, $\left.h_{n} j_{n}\right)$ \} defined on $\bar{\Omega}_{h}$ equipped with the norm

$$
\left\|\varphi^{h}\right\|_{L_{2 h}}=\left(\sum_{x \in \Omega_{h}}\left|\varphi^{h}(x)\right|^{2} h_{1} \cdots h_{n}\right)^{\frac{1}{2}} .
$$

To the differential operator $A^{x}$ defined by (18), we assign the difference operator $A_{h}^{x}$ by the formula

$$
A_{h}^{x} u^{h}=-\sum_{r=1}^{n}\left(\alpha_{r}(x) u_{x_{r}}^{h}\right)_{x_{r}, j_{r}},
$$


where $A_{h}^{x}$ is known as self-adjoint positive definite operator in $L_{2 h}$, acting in the space of grid functions $u^{h}(x)$ satisfying the conditions $u^{h}(x)=0$ for all $x \in S_{h}$. With the help of the difference operator $A_{h}^{x}$, we arrive at the following boundary value problem:

$$
\left\{\begin{array}{c}
u_{t t}^{h}(t, x)+\alpha u_{t}^{h}(t, x)+A_{h}^{x} u^{h}(t, x)+\beta u^{h}(t, x)=f^{h}(t, x), \\
0<t<T, x \in \Omega_{h}, \\
u^{h}(0, x)=\varphi^{h}(x), \quad u_{t}^{h}(0, x)=\psi^{h}(x), \quad x \in \Omega_{h} .
\end{array}\right.
$$

In the second step, we replace (41) with the difference scheme (19)

$$
\left\{\begin{array}{l}
\frac{u_{k+1}^{h}(x)-2 u_{k}^{h}(x)+u_{k-1}^{h}(x)}{\tau^{2}}+\alpha \frac{u_{k+1}^{h}(x)-u_{k-1}^{h}(x)}{2 \tau}+A_{h}^{x} u_{k+1}^{h}(x)+\beta u_{k+1}^{h}(x)=f_{k}^{h}(x), \\
f_{k}^{h}(x)=f^{h}\left(t_{k+1}, x\right), t_{k}=k \tau, 1 \leq k \leq N-1, x \in \Omega_{h}, N \tau=T, \\
u_{0}^{h}(x)=\varphi^{h}(x), \\
\frac{u_{1}^{h}(x)-u_{0}^{h}(x)}{\tau}+\left(A_{h}^{x}+\left(\beta-\frac{\alpha^{2}}{4}\right) I_{h}\right) \tau u_{1}^{h}(x)=\frac{1}{1+\frac{\alpha}{2} \tau} \psi^{h}(x), \quad x \in \Omega_{h}
\end{array}\right.
$$

for an infinite system of ordinary differential equations.

Theorem 3.4 For the solution $\left\{u_{k}^{h}(x)\right\}_{0}^{N}$ of problem (38) the following stability estimates:

$$
\begin{aligned}
& \max _{1 \leq k \leq N}\left\|u_{k}^{h}\right\|_{L_{2 h}} \leq M_{1}\left\{\max _{1 \leq k \leq N-1}\left\|f_{k}^{h}\right\|_{L_{2 h}}+\left\|\psi^{h}\right\|_{L_{2 h}}+\left\|\varphi^{h}\right\|_{L_{2 h}}\right\} \\
& \max _{1 \leq k \leq N}\left\|u_{k}^{h}\right\|_{W_{2 h}^{1}} \leq M_{1}\left\{\max _{1 \leq k \leq N-1}\left\|f_{k}^{h}\right\|_{L_{2 h}}+\left\|\psi^{h}\right\|_{L_{2 h}}+\left\|\varphi^{h}\right\|_{W_{2 h}^{1}}\right\} \\
& \max _{1 \leq k \leq N}\left\|u_{k}^{h}\right\|_{W_{2 h}^{2} \leq} \leq M_{2}\left\{\max _{2 \leq k \leq N-1}\left\|\frac{1}{\tau}\left(f_{k}^{h}-f_{k-1}^{h}\right)\right\|_{L_{2 h}}\right. \\
&\left.+\left\|f_{1}^{h}\right\|_{L_{2 h}}+\left\|\psi^{h}\right\|_{W_{2 h}^{1}}+\left\|\varphi^{h}\right\|_{W_{2 h}^{2}}\right\}
\end{aligned}
$$

hold, where $M_{1}$ and $M_{2}$ do not depend on $\varphi^{h}(x), \psi^{h}(x)$ and $f_{k}^{h}(x), 1 \leq k \leq N-1$.

Proof Difference scheme (38) can be written in abstract form (39) in a Hilbert space $L_{2 h}=$ $L_{2}\left(\bar{\Omega}_{h}\right)$ with self-adjoint positive definite operator $A_{h}=A_{h}^{x}$ by formula (40).

Here, $f_{k}^{h}=f_{k}^{h}(x)$ and $u_{k}^{h}=u_{k}^{h}(x)$ are known and unknown abstract mesh functions defined on $\bar{\Omega}_{h}$ with the values in $H=L_{2 h}$. Therefore, estimates of Theorem 3.4 follow from estimates (22), (23), and (24) and the following theorem on the coercivity inequality for the solution of the elliptic difference problem in $L_{2 h}$.

Theorem 3.5 For the solutions of the elliptic difference problem [26]

$$
\left\{\begin{array}{l}
A_{h}^{x} u^{h}(x)=\omega^{h}(x), \quad x \in \Omega_{h} \\
u^{h}(x)=0, \quad x \in S_{h}
\end{array}\right.
$$

the following coercivity inequality holds:

$$
\sum_{r=1}^{n}\left\|u_{x_{r} x_{r}}^{h}\right\|_{L_{2 h}} \leq M_{3}\left\|\omega^{h}\right\|_{L_{2 h}}
$$

where $M_{3}$ does not depend on $h$ and $\omega^{h}$. 
Note that the difference schemes of the second order of accuracy with respect to one variable for approximate solutions of the mixed problems (10) and (15) generated by the difference schemes (34) and (35) can be constructed. The abstract theorem given above and Theorem 3.5 permit us to establish the stability estimates for the solution of these difference schemes.

In applications, one test example is considered. The theoretical statements for the solution of these difference schemes are supported by the result of the numerical experiment.

\section{Numerical results}

In applications, the theorems on convergence estimates can be established. The theoretical statements for the solution of difference schemes can be supported by the result of the numerical experiment. We have not been able to obtain a sharp estimate for the constants figuring in the stability inequality. Therefore we will give the results of numerical experiments for the initial-boundary value problem:

$$
\left\{\begin{array}{l}
\frac{\partial^{2} u(t, x)}{\partial t^{2}}+2 \frac{\partial u(t, x)}{\partial t}-\frac{\partial^{2} u(t, x)}{\partial x^{2}}+u(t, x)=e^{-t} \sin x \\
0<t<1,0<x<\pi \\
u(0, x)=\sin x, \quad \frac{\partial}{\partial t} u(0, x)=-\sin x, \quad 0 \leq x \leq \pi \\
u(t, 0)=u(t, \pi)=0, \quad 0 \leq t \leq 1
\end{array}\right.
$$

for the telegraph equation. The exact solution of this problem is

$$
u(t, x)=e^{-t} \sin x .
$$

For the approximate solution of the initial-boundary value problem (44), we consider the set $w_{\tau, h}=[0,1]_{\tau} \times[0, \pi]_{h}$ of a family of grid points depending on the small parameters $\tau$ and $h$. We present the following difference scheme of the first order of accuracy in $t$ and second order of accuracy in $x$ for the approximate solutions of the problem (44):

$$
\left\{\begin{array}{l}
\frac{u_{n}^{k+1}-2 u_{n}^{k}+u_{n}^{k-1}}{\tau^{2}}+2 \frac{u_{n}^{k+1}-u_{n}^{k-1}}{2 \tau}-\frac{u_{n+1}^{k+1}-2 u_{n}^{k+1}+u_{n-1}^{k+1}}{h^{2}}+u_{n}^{k+1} \\
\quad=e^{-t_{k+1}} \sin x_{n}, \quad x_{n}=n h, t_{k+1}=(k+1) \tau, 1 \leq k \leq N-1,1 \leq n \leq M-1, \\
u_{n}^{0}=\sin x_{n}, \quad \frac{u_{n}^{1}-u_{n}^{0}}{\tau}=-\sin x_{n}, \quad 0 \leq n \leq M, \\
u_{0}^{k}=u_{M}^{k}=0, \quad 0 \leq k \leq N .
\end{array}\right.
$$

Now, we consider two types of difference schemes of second order of accuracy in $t$ and $x$ for the approximate solutions of the problem (44):

$$
\left\{\begin{array}{l}
\frac{u_{n}^{k+1}-2 u_{n}^{k}+u_{n}^{k-1}}{\tau^{2}}+2 \frac{u_{n}^{k+1}-u_{n}^{k-1}}{2 \tau}-\frac{1}{2} \frac{u_{n+1}^{k+1}-2 u_{n}^{k+1}+u_{n-1}^{k+1}}{h^{2}}-\frac{1}{2} \frac{u_{n+1}^{k-1}-2 u_{n}^{k-1}+u_{n-1}^{k-1}}{h^{2}}+\frac{1}{2}\left(u_{n}^{k+1}+u_{n}^{k-1}\right) \\
\quad=e^{-t_{k}} \sin \left(x_{n}\right), \quad x_{n}=n h, t_{k}=k \tau, 1 \leq k \leq N-1,1 \leq n \leq M-1, \\
u_{n}^{0}=\sin \left(x_{n}\right), \quad x_{n}=n h, \\
\frac{u_{n}^{1}-u_{n}^{0}}{\tau}=-\sin \left(x_{n}\right)+\frac{\tau}{2} \frac{u_{n}^{2}-2 u_{n}^{1}+u_{n}^{0}}{\tau^{2}}, \quad 0 \leq n \leq M, \\
u_{0}^{k}=u_{M}^{k}=0, \quad 0 \leq k \leq N,
\end{array}\right.
$$


Table 1 Error analysis

$$
\begin{aligned}
& \begin{array}{llll}
\hline \boldsymbol{\tau}=\frac{\mathbf{1}}{\boldsymbol{N}}, \boldsymbol{h}=\frac{\boldsymbol{p i}}{\boldsymbol{M}} & \boldsymbol{N}=\boldsymbol{M}=\mathbf{2 0} & \boldsymbol{N}=\boldsymbol{M}=\mathbf{4 0} & \boldsymbol{N}=\boldsymbol{M}=\mathbf{8 0} \\
\hline \text { The difference scheme (45) } & 7.9931 \times 10^{-4} & 4.2932 \times 10^{-4} & 2.2201 \times 10^{-4} \\
\text { The difference scheme (46) } & 2.3651 \times 10^{-4} & 6.0209 \times 10^{-5} & 1.5196 \times 10^{-5} \\
\text { The difference scheme (47) } & 1.3510 \times 10^{-4} & 3.4524 \times 10^{-5} & 8.7409 \times 10^{-6} \\
\hline
\end{array} \\
& \left\{\begin{array}{l}
\frac{u_{n}^{k+1}-2 u_{n}^{k}+u_{n}^{k-1}}{\tau^{2}}+2 \frac{u_{n}^{k+1}-u_{n}^{k-1}}{2 \tau}-\frac{1}{2} \frac{u_{n+1}^{k}-2 u_{n}^{k}+u_{n-1}^{k}}{h^{2}}-\frac{1}{4} \frac{u_{n+1}^{k+1}-2 u_{n}^{k+1}+u_{n-1}^{k+1}}{h^{2}} \\
\quad-\frac{1}{4} \frac{u_{n+1}^{k-1}-2 u_{n}^{k-1}+u_{n-1}^{k-1}}{h^{2}}+\frac{1}{2} u_{n}^{k}+\frac{1}{4}\left(u_{n}^{k+1}+u_{n}^{k-1}\right) \\
\quad=e^{-t_{k}} \sin \left(x_{n}\right), \quad x_{n}=n h, t_{k}=k \tau, 1 \leq k \leq N-1,1 \leq n \leq M-1, \\
u_{n}^{0}=\sin \left(x_{n}\right), \quad x_{n}=n h, \\
\frac{u_{n}^{1}-u_{n}^{0}}{\tau}=-\sin \left(x_{n}\right)+\frac{\tau}{2} \frac{u_{n}^{2}-2 u_{n}^{1}+u_{n}^{0}}{\tau^{2}}, \quad 0 \leq n \leq M, \\
u_{0}^{k}=u_{M}^{k}=0, \quad 0 \leq k \leq N .
\end{array}\right.
\end{aligned}
$$

To solve these difference equations, a modified Gauss elimination method procedure is applied. Hence, we seek a solution of the matrix equation in the following form:

$$
u_{j}=\alpha_{j+1} u_{j+1}+\beta_{j+1}, \quad u_{M}=0, \quad j=M-1, \ldots, 2,1,
$$

where $\alpha_{j}(j=1,2, \ldots, M)$ are $(N+1) \times(N+1)$ square matrices, and $\beta_{j}(j=1,2, \ldots, M)$ are $(N+1) \times 1$ column matrices defined by

$$
\begin{aligned}
& \alpha_{j+1}=-\left(B+C \alpha_{j}\right)^{-1} A, \\
& \beta_{j+1}=\left(B+C \alpha_{j}\right)^{-1}\left(D \phi-C \beta_{j}\right), \quad j=1,2, \ldots, M-1,
\end{aligned}
$$

where $j=1,2, \ldots, M-1, \alpha_{1}$ is the $(N+1) \times(N+1)$ zero matrix, and $\beta_{1}$ is the $(N+1) \times 1$ zero matrix. The results of computer calculations show that the second-order difference schemes are more accurate than the difference scheme of the first order of accuracy. Table 1 is constructed for $N=M=20,40$, and 80 , respectively.

The errors are computed by

$$
E_{M}^{N}=\max _{1 \leq k \leq N-1,1 \leq n \leq M-1}\left|u\left(t_{k}, x_{n}\right)-u_{n}^{k}\right|,
$$

where $u\left(t_{k}, x_{n}\right)$ represents the exact solution and $u_{n}^{k}$ represents the numerical solution at $\left(t_{k}, x_{n}\right)$ and the results are given in Table 1.

\section{Conclusion}

In the present paper, we have discussed the Cauchy problem (1) for the abstract telegraph equations. Stability estimates for the solution of this problem are established. The difference schemes of the first order and second order of accuracy for telegraph equations are studied. The stability of the difference schemes is established. One test example is given and numerical results are compared with the exact solution. The comparison convinces us that the finite difference scheme method of the second order of approximation gives better results. Numerical results are obtained using Matlab. The theoretical statements for the solution of these difference schemes are supported by the numerical results. Moreover, applying the result of the monograph [3], the nonlocal boundary value problem for this 
abstract telegraph equations can be investigated. Of course, the stability estimates for the solution of the nonlocal boundary value problem have been established. The difference schemes of the first order and second order of accuracy for telegraph equations can be studied. The stability of the difference schemes has been established without any assumptions as regards the grid steps.

\section{Competing interests}

The authors declare that they have no competing interests.

Authors' contributions

All authors read and approved the final manuscript.

\section{Author details}

'Department of Mathematics, Fatih University, Istanbul, 34500, Turkey. ${ }^{2}$ Department of Mathematics, Siirt University, Siirt, 56100 , Turkey.

\section{Acknowledgements}

The authors are very grateful to the referees for their valuable and helpful comments, remarks, and suggestions.

Received: 19 November 2014 Accepted: 4 February 2015 Published online: 24 February 2015

\section{References}

1. Fattorini, HO: Second Order Linear Differential Equations in Banach Spaces. North-Holland, Amsterdam (1985)

2. Goldstein, JA: Semigroups of Linear Operators and Applications. Oxford Mathematical Monographs. Oxford University Press, New York (1985)

3. Ashyralyev, A, Sobolevskii, PE: New Difference Schemes for Partial Differential Equations. Operator Theory: Advances and Applications. Birkhäuser, Basel (2004)

4. Ashyralyev, A, Sobolevskii, PE: Well-Posedness of Parabolic Difference Equations. Operator Theory: Advances and Applications. Birkhäuser, Basel (1994)

5. Ghorbanalizadeh, A, Sawano, Y: Approximation in Banach space by linear positive operators. Positivity 18(3), 585-594 (2014)

6. Ashyralyev, A, Sobolevskii, PE: Two new approaches for construction of the high order of accuracy difference schemes for hyperbolic differential equations. Discrete Dyn. Nat. Soc. 2005(2), 183-213 (2005)

7. De la Sen, M: On bounded strictly positive operators of closed range and some applications to asymptotic hyperstability of dynamic systems. Abstr. Appl. Anal. 2013, Article ID 639576 (2013). doi:10.1155/2013/639576

8. De la Sen, M: Positivity and stability of the solutions of Caputo fractional linear time-invariant systems of any order with internal point delays. Abstr. Appl. Anal. 2011, Article ID 161246 (2011). doi:10.1155/2011/161246

9. Achour, D, Belacel, A: Domination and factorization theorems for positive strongly $p$-summing operators. Positivity 18(4), 785-804 (2014)

10. Krein, SG: Linear Differential Equations in Banach Space. Translations of Mathematical Monographs, vol. 29. Am. Math Soc., Providence (1971); Translated from the Russian by JM Danskin

11. Vasilev, W, Krein, SG, Piskarev, S: Operator semigroups, cosine operator functions, and linear differential equations. In: Mathematical Analysis. Itogi Nauki i Tekhniki, vol. 28, pp. 87-202. Akad. Nauk SSSR Vsesoyuz. Inst. Nauchn. i Tekhn. Inform., Moscow (1990) (Russian); Translated in J. Soviet Math. 54(4), 1042-1129 (1991)

12. Jordan, PM, Puri, A: Digital signal propagation in dispersive media. J. Appl. Phys. 85(3), 1273-1282 (1999)

13. Weston, $\mathrm{VH}, \mathrm{He}, \mathrm{S}$ : Wave splitting of telegraph equation in $\mathrm{R}^{3}$ and its applications to inverse scattering. Inverse Probl. 9, 789-812 (1993)

14. Banasiak, J, Mika, JR: Singularly perturbed telegraph equations with applications in the random walk theory. J. Appl. Math. Stoch. Anal. 11(1), 9-28 (1998)

15. Dehghan, M, Shokri, A: A numerical methods for solving the hyperbolic telegraph equation. Numer. Methods Partial Differ. Equ. 24(4), 1080-1093 (2008)

16. Gao, F, Chi, C: Unconditionally stable difference schemes for a one-space-dimensional linear hyperbolic equation. Appl. Math. Comput. 187(2), 1272-1276 (2007)

17. Biazar, J, Ebrahimi, H, Ayati, Z: An approximation to the solution of telegraph equation by variational iteration method. Numer. Methods Partial Differ. Equ. 25, 797-801 (2009)

18. Saadatmandi, A, Dehghan, M: Numerical solution of hyperbolic telegraph equation using the Chebyshev tau method. Numer. Methods Partial Differ. Equ. 26(1), 239-252 (2010)

19. Koksal, ME: An operator-difference method for telegraph equations arising in transmission lines. Discrete Dyn. Nat. Soc. 2011, Article ID 561015 (2011). doi:10.1155/2011/561015

20. Twizell, EH: An explicit difference method for the wave equation with extended stability range. BIT Numer. Math. 19(3), 378-383 (1979)

21. Ashyralyev, A, Akat, M: An approximation of stochastic hyperbolic equations. AIP Conf. Proc. 1389, 625-628 (2011)

22. Ashyralyev, A, Akat, M: An approximation of stochastic hyperbolic equations: case with Wiener process. Math. Methods Appl. Sci. 36(9), 1095-1106 (2013)

23. Ashyralyev, A, Akat, M: An approximation of stochastic telegraph equations. AlP Conf. Proc. 1479, 598-601 (2012)

24. Pogorelenko, V, Sobolevskii, PE: The 'counter-example' to W. Littman counter-example of $L_{p}$-energetical inequality for wave equation. Funct. Differ. Equ. 4(1-2), 165-172 (1997)

25. Kostin, VA: Analytic semigroups and cosine functions. Dokl. Akad. Nauk SSSR 307(4), 796-799 (1989) (Russian)

26. Sobolevskii, PE: Difference Methods for the Approximate Solution of Differential Equations. Izdat. Voronezh. Gosud. Univ., Voronezh (1975) (Russian) 